Applied Mathematical Sciences, Vol. 10, 2016, no. 62, 3075 - 3085

HIKARI Ltd, www.m-hikari.com

https://doi.org/10.12988/ams.2016.67219

\title{
Testing for Correlation between Survival Probabilities: an Analytically Tractable Stochastic Model
}

\author{
Maria Cristina Recchioni \\ Dipartimento di Management \\ Università Politecnica delle Marche \\ Piazzale Martelli 8, 60121 Ancona, Italy \\ Adina Scoccia \\ Dipartimento di Management \\ Università Politecnica delle Marche \\ Piazzale Martelli 8, 60121 Ancona, Italy
}

Copyright (c) 2016 Maria Cristina Recchioni and Adina Scoccia. This article is distributed under the Creative Commons Attribution License, which permits unrestricted use, distribution, and reproduction in any medium, provided the original work is properly cited.

\begin{abstract}
A stochastic model to describe the joint dynamics of financial market variables (i.e., equity market index, gross domestic product) and survival probability is proposed. The model is analytically tractable and may be used to price some mortality derivatives. A simulation study is carried out.
\end{abstract}

Keywords: Stochastic volatility model, survival probability, Kolmogorov backward equation, calibration procedure

\section{Introduction}

Mortality is a major risk facing large re-insurers. Assets traded in the financial markets are used to pay mortality claims, so analysis of the correlation between assets and mortality rate is an interesting issue. In fact, a severe pandemic (such as Ebola virus disease) could strongly affect the economy and/or financial markets, generating a depreciation of assets and inducing a crisis for companies 
using these assets to pay mortality claims (see Dacorogna, M, Cadena, M (2015)). Indeed, some countries in the Euro zone have recently registered an increase in the mortality rate, which is probably due to the impact of the economic crisis on low-income families.

This paper contributes to this analysis by introducing a stochastic volatility model to describe the joint dynamics of mortality rate and asset price while admitting an indirect correlation between the two.

The proposed model is based on the mortality model illustrated in MilevskyPromislow 2001 and on the generalized Heston model of Recchioni and Scoccia 2014. The model is analytically tractable in that it allows us to deduce an integral representation formula for the transition probability density function. The most relevant formulas are proved using an approach similar to those illustrated in Da Fonseca et al. 2008, Fatone et al. 2009, 2013, Fouque et al. 2003, and Recchioni and $\mathrm{Yu}, 2016$.

The proposed model may not be included in the class of affine models such as those proposed by Biffis 2005, Schrager 2006, Luciano and Vigna 2008, Blackburn and Sherris 2013 although it is still analytically tractable.

Moreover, the indirect correlation between asset and mortality rate introduced here permits the study of how the profound crisis in western countries (measured by some financial and/or economic index) has affected the mortality rate. A similar study can be found in Denuit 2009, where the relationship between some financial asset prices and baby-boomer retirement is analyzed in order to give empirical evidence that financial asset prices are likely to fall as baby boomers retire. As well, the numerical results illustrated in Jalen and Mamon 2009 show that the dependence between mortality and financial factors plays a crucial role in pricing insurance contracts with long-term maturities.

This paper is organized as follows. In Section 2 we present the time continuous stochastic model which describes the joint dynamics of the asset/market index and the mortality rate and some relevant formulas. In Section 3 we deduce the formula for transition probability density function and we illustrate some expansions. Finally, in Section 4 we illustrate a simulation study which highlights some relevant features of the proposed model.

\section{The joint dynamics of asset price and mor- tality rate}

We present the model describing the joint dynamics of the index/asset price $S_{t}$ and the mortality rate $h_{t}$. The mortality rate, also known as the hazard rate, represents the instantaneous rate of mortality of a certain population measured on an annualized basis. The population we have in mind is a human population made of individuals belonging to a cohort, that is, the population 
of individuals born in a given year.

Following Milevsky-Promislow 2001, the mortality rate, $h_{t}$, is defined by the so-called mean reverting Brownian Gompertz specification:

$$
h_{t}=h_{0} e^{g t+\sigma y_{t}},
$$

and

$$
d y_{t}=-b y_{t} d t+d B_{t},
$$

where $B_{t}$ is a Wiener process. $B_{0}=0, d B_{t}$ denotes the stochastic differential of $B_{t}$, and $b, g, h_{0}$ are positive parameters. In contrast to Milevsky-Promislow 2001 , this paper correlates the dynamics of the variable $y_{t}$ to the dynamics of the asset/index price modifying Eq. (2):

$$
\begin{aligned}
d S_{t} & =S_{t} \mu d t+S_{t} \sqrt{v_{t}} d W_{t}+S_{t} a d Q_{t} \\
d v_{t} & =\chi\left(\theta-v_{t}\right) d t+\varepsilon \sqrt{v_{t}} d Z_{t}
\end{aligned}
$$

and

$$
d y_{t}=-b y_{t} d t+d B_{t}+\gamma d Q_{t}
$$

where $\mu, a, \chi, \theta, \varepsilon, \gamma$ are suitable real constants satisfying the following conditions:

$$
\begin{aligned}
a, \chi, \theta, \varepsilon & >0, \\
\frac{2 \chi \theta}{\varepsilon^{2}} & >1,
\end{aligned}
$$

and $W_{t}, Z_{t}, Q_{t}$ are standard Wiener processes such that $W_{0}=Z_{0}=0$, while $d W_{t}, d Z_{t}, d Q_{t}$ are the stochastic differentials of the Wiener processes just mentioned. All correlations among Wiener processes are zero with the exception of the following:

$$
\begin{aligned}
& E\left(d W_{t} d Z_{t}\right)=\rho_{v} d t, \\
& E\left(d Q_{t} d B_{t}\right)=\rho_{Y} d t,
\end{aligned}
$$

where $E(\cdot)$ denotes the expected value of $\cdot$ and $\rho_{v}, \rho_{Y} \in(-1,1)$ are the correlation coefficients, which we assume to be constant. Under condition (7), the variance $v_{t}$ is positive with probability one for any $t>0$ given that $v_{0}$ is positive with probability one.

In the stochastic model (1), (3)-(5) the asset price process $S_{t}$ and the mortality rate $h_{t}$ are indirectly correlated via the process $y_{t}$. The specific form of model (1), (3)-(5) allows us to derive a semi-explicit formula for the transition probability density function of the process $\left(x_{t}, v_{t}, y_{t}\right), t>0$, where $x_{t}$ is the logarithm of the price, that is, $x_{t}=\log S_{t}$. 
Using Ito's lemma, the dynamical system (3)-(5) can be rewritten as follows:

$$
\begin{aligned}
d x_{t} & =\left(\mu-\frac{1}{2} a^{2}-\frac{1}{2} v_{t}\right) d t+\sqrt{v_{t}} d W_{t}+a d Q_{t}, t>0 \\
d v_{t} & =\chi\left(\theta-v_{t}\right) d t+\varepsilon \sqrt{v_{t}} d Z_{t} \\
d y_{t} & =-b y_{t} d t+d B_{t}+\gamma d Q_{t} .
\end{aligned}
$$

Equations (10)-(12) must be equipped with initial conditions as follows:

$$
\begin{aligned}
x_{0} & =\tilde{x}_{0}, \\
v_{0} & =\tilde{v}_{0}, \\
Y_{0} & =\tilde{Y}_{0}=0,
\end{aligned}
$$

where $\tilde{x}_{0}, \tilde{v}_{0}$ and $\tilde{Y}_{0}$ are random variables that we assume to be concentrated in a point with probability one. For simplicity, we identify the random variables $\tilde{x}_{0}, \tilde{v}_{0}$ and $\tilde{Y}_{0}$ with the points where they are concentrated.

Let $\mathbb{R}^{+}$be the set of the positive real numbers and $\mathbb{R}$ be real Euclidean space, while $(x, v, y, t)$ and $\left(x^{\prime}, v^{\prime}, y^{\prime}, t^{\prime}\right), t<t^{\prime}$ are the variables in the past and the future, respectively, with $(x, v, y, t)$ and $\left(x^{\prime}, v^{\prime}, y^{\prime}, t^{\prime}\right) \in \mathbb{R} \times \mathbb{R}^{+} \times \mathbb{R} \times \mathbb{R}^{+}$. The transition probability density function, $p_{f}\left(x, v, y, t, x^{\prime}, v^{\prime}, y^{\prime}, t^{\prime}\right)$, associated with the stochastic process implicitly defined by $(10)-(12)$ is the solution to the Kolmogorov backward equation regarded as a function of the past variables $(x, v, y, t) \in \mathbb{R} \times \mathbb{R}^{+} \times \mathbb{R} \times \mathbb{R}^{+}:$

$$
\begin{gathered}
-\frac{\partial p_{f}}{\partial t}=\frac{1}{2}\left(v+a^{2}\right) \frac{\partial^{2} p_{f}}{\partial x^{2}}+\frac{1}{2} \epsilon^{2} v \frac{\partial^{2} p_{f}}{\partial v^{2}}+\epsilon v \rho_{v} \frac{\partial^{2} p_{f}}{\partial x \partial v} \\
+a\left(\gamma+\rho_{Y}\right) \frac{\partial^{2} p_{f}}{\partial x \partial y}+\frac{\varphi^{2}}{2} \frac{\partial^{2} p_{f}}{\partial y^{2}}+\chi(\theta-v) \frac{\partial p_{f}}{\partial v}-b y \frac{\partial p_{f}}{\partial y}+\left(\mu-\frac{1}{2} a^{2}-\frac{1}{2} v\right) \frac{\partial p_{f}}{\partial x}
\end{gathered}
$$

with suitable boundary conditions and final condition:

$$
p_{f}\left(x, v, y, t^{\prime}, x^{\prime}, v^{\prime}, y^{\prime}, t^{\prime}\right)=\delta\left(x-x^{\prime}\right) \delta\left(v-v^{\prime}\right) \delta\left(y-y^{\prime}\right) .
$$

In Eqs. (16), (17), $\delta(\cdot)$ denotes the Dirac delta function and $\varphi$ is given by:

$$
\varphi=\sqrt{\left(\gamma+\rho_{Y}\right)^{2}+\left(1-\rho_{Y}^{2}\right)}
$$

As shown in Section 3, the following integral representation formula for $p_{f}$ holds:

$$
\begin{gathered}
p_{f}\left(x, v, y, t, x^{\prime}, v^{\prime}, y^{\prime}, t^{\prime}\right)=\frac{1}{2 \pi} \int_{\mathbb{R}} d k e^{\imath k\left[\left(x^{\prime}-x\right)-\left(t^{\prime}-t\right)\left(\mu-\frac{1}{2} a^{2}\right)\right]} e^{-a^{2} k^{2}\left(t-t^{\prime}\right) / 2} \times \\
H\left(v, v^{\prime}, k, t^{\prime}-t\right) \frac{1}{\varphi \sqrt{2 \pi \psi_{2}\left(t^{\prime}-t\right)}} e^{-\frac{1}{2 \varphi^{2} \psi_{2}\left(t^{\prime}-t\right)}\left(y^{\prime}-y e^{-b\left(t^{\prime}-t\right)}+\imath k a\left(\gamma+\rho_{Y}\right) \psi_{1}\left(t^{\prime}-t\right)\right)^{2}}
\end{gathered}
$$


where $H$ is the function given by

$$
\begin{aligned}
& H\left(v, v^{\prime}, k, \tau\right)=M e^{-M\left(\tilde{v}+v^{\prime}\right)}\left(\frac{v^{\prime}}{\tilde{v}}\right)^{\left(\chi \theta / \varepsilon^{2}\right)-1 / 2} I_{2 \chi \theta / \varepsilon^{2}-1}\left(2 M(\tilde{v} v)^{1 / 2}\right) \times \\
& e^{-\frac{2 \chi \theta}{\varepsilon^{2}}(\nu+\zeta) \tau-\frac{2 \chi \theta}{\varepsilon^{2}} \ln \left(s_{\beta} /(2 \zeta)\right)} e^{-2 v\left(\zeta^{2}-\nu^{2}\right) s_{\gamma} /\left(\varepsilon^{2} s_{\beta}\right)}, \quad \tau=t^{\prime}-t>0,
\end{aligned}
$$

$I_{q}$ is the modified Bessel function of order $q$ (see, for example, Abramowitz and Stegun, 1970) and the quantities $\psi_{j}, j=1,2, \nu, \zeta, s_{\beta}, s_{\gamma}, \tilde{v}$, and $M$, are given by

$$
\begin{gathered}
\psi_{j}(\tau)=\frac{1-e^{-j b \tau}}{j b}, \quad j=1,2, \\
\nu=-\frac{1}{2}\left(\chi+\imath k \varepsilon \rho_{v}\right), \\
\zeta=\frac{1}{2}\left(4 \nu^{2}+\varepsilon^{2}\left(k^{2}-\imath k\right)\right)^{1 / 2}, \\
s_{\gamma}=1-e^{-2 \zeta \tau}, s_{\beta}=(\zeta-\nu)+(\zeta+\nu) e^{-2 \zeta \tau}, \tau=t^{\prime}-t>0, \\
\tilde{v}=\frac{4 v \zeta^{2} e^{-2 \zeta \tau}}{\left(s_{\beta}\right)^{2}}, M=\frac{2 s_{\beta}}{\varepsilon^{2} s_{\gamma}}, \tau=t^{\prime}-t>0 .
\end{gathered}
$$

\section{Derivation of the integral representation for- mula for the transition probability density function}

Theorem 1 The transition probability density function, $p_{f}$, of the stochastic process $\left(x_{t}, v_{t}, y_{t}\right)$ defined by (10)-(12) is given by formula (19).

This section is devoted to the proof the Theorem 1 . With $\tau=t^{\prime}-t$ and due to the fact that the coefficients in Eqs. (16) and (17) are independent of time and $x$ translations, we seek the solution of Eqs. (16) and (17) in the form

$$
\begin{aligned}
& p_{f}\left(x, v, y, t, x^{\prime}, v^{\prime}, y^{\prime}, t^{\prime}\right)= \\
& \frac{1}{(2 \pi)^{3}} \int_{\mathbb{R}} e^{\imath k\left(x^{\prime}-x\right)} \int_{\mathbb{R}} e^{\imath l v^{\prime}} \int_{\mathbb{R}} e^{\imath \xi y^{\prime}} f(\tau, v, y, k, l, \xi) d \xi d l d k .
\end{aligned}
$$

Here, $f$ is the Fourier transform with respect to the "future" variables of the function obtained by extending $p_{f}$ as a function of these "future" variables $\left(x^{\prime}, v^{\prime}, y^{\prime}\right) \in \mathbb{R} \times \mathbb{R}^{+} \times \mathbb{R}$, with zero when $v^{\prime} \notin \mathbb{R}^{+}$, while $k, l, \xi$, are the conjugate variables in the Fourier transform of $x^{\prime}-x, v^{\prime}$ and $y^{\prime}$. Using the fact that $-\frac{\partial}{\partial t}=\frac{\partial}{\partial \tau}$ and Eqs. (16), (17) and (26) it is easy to see that $f$ satisfies the initial condition

$$
f(0, v, y, k, l, \xi)=e^{-\imath \xi y} e^{-\imath l v}
$$


Since the coefficients of the partial differential operator appearing on the right hand side of (16) and the initial condition (27) are first degree polynomials in $v$ and $y$, we seek a solution of problem (16), (27) of the form:

$$
f(\tau, v, y, k, l, \xi)=e^{-v B(\tau, k, l)} e^{-y Q(\tau, k, \xi)} e^{A(\tau, k, l, \xi)}
$$

where $A, B$, and $Q$ are functions to be determined. Substituting formula (26) into equation (16), we obtain that functions $A, B$, and $Q$ satisfy the following ordinary differential equations:

$$
\begin{gathered}
\frac{d A}{d \tau}(\tau, k, l, \xi)=-\frac{a^{2}}{2} k^{2}-\imath k\left(\mu-\frac{1}{2} a^{2}\right) \\
-\chi \theta B(\tau, k, l)+\frac{\varphi^{2}}{2} Q^{2}(\tau, k, \xi)+\imath a\left(\gamma+\rho_{Y}\right) k Q(\tau, k, \xi), \\
\frac{d B}{d \tau}(\tau, k, l)=-\frac{\varepsilon^{2}}{2} B^{2}-\left(\imath k \varepsilon \rho_{v}+\chi\right) B+\frac{1}{2}\left(k^{2}-\imath k\right), \\
\frac{d Q}{d \tau}(\tau, k, \xi)=-b Q
\end{gathered}
$$

with initial conditions:

$$
A(0, k, l, \xi)=0, \quad B(0, k, l)=\imath l, \quad Q(0, k, \xi)=\imath \xi .
$$

Note that initial condition (32) has been obtained by imposing (27) on the function $f$ given by (28). Equations (30) and (31) are Riccati equations. Integrating (29) with respect to $\tau$ we obtain

$$
B(\tau, k, l)=\frac{2}{\varepsilon^{2}} \frac{\frac{d C(\tau, k, l)}{d \tau}}{C(\tau, k, l)}=\frac{2}{\varepsilon^{2}} \frac{(\nu-\zeta)(\nu+\zeta-\imath l) e^{-2 \zeta \tau}+(\nu+\zeta)(\imath l-\nu+\zeta)}{(\nu+\zeta-\imath l) e^{-2 \zeta \tau}+(\imath l-\nu+\zeta)},
$$

where $\nu, \zeta$ are given by (22), (23), $C$ is given by

$$
C(\tau, k, l)=\frac{e^{(\nu-\zeta) \tau}}{2 \zeta}\left[(\nu+\zeta-\imath l) e^{-2 \zeta \tau}+(\imath l-\nu+\zeta)\right]
$$

$Q$ and $A$ are as follows:

$$
\begin{gathered}
Q(\tau, k, \xi)=\imath \xi e^{-b \tau}, \\
A(\tau, k, l, \xi)=-\tau \frac{a^{2}}{2} k^{2}-\imath k \tau\left(\mu-\frac{1}{2} a^{2}\right)-a\left(\gamma+\rho_{Y}\right) \psi_{1}(\tau) k \xi \\
-\frac{\varphi^{2} \psi_{2}(\tau)}{2} \xi^{2}-\frac{2 \chi \theta}{\epsilon^{2}} \ln C(\tau, k, l) .
\end{gathered}
$$


Substituting equations (33), (34) and (36) into equation (26), integrating with respect to $l$ (see, for example, Oberhettinger, 1973) and using the following formula:

$$
\begin{aligned}
& \frac{1}{2 \pi} \int_{-\infty}^{+\infty} e^{\imath \xi y^{\prime}} e^{-\imath \xi y e^{-b \tau}} e^{\imath \xi k a\left(\gamma+\rho_{Y}\right) \psi_{1}(\tau)-\xi^{2} \gamma^{2} \psi_{2}(\tau) / 2} d \xi= \\
& \frac{1}{\varphi \sqrt{2 \pi \psi_{2}(\tau)}} e^{-\frac{1}{2 \varphi^{2} \psi_{2}(\tau)}\left(y^{\prime}-y e^{-b \tau}+\imath k a\left(\gamma+\rho_{Y}\right) \psi_{1}(\tau)\right)^{2}},
\end{aligned}
$$

we obtain formula (19).

\section{Simulation study}

Model (1), (3)-(5) proposed in this paper can be applied to price financial products which depend on a financial index and the residual life of the owner, such as index linked insurance policies. In this section we just simulate model (1), (3)-(5) to illustrate the effects of the indirect correlation between asset and mortality rate on the probability, $\mathcal{S}_{n}$, that an individual aged $n$ in the current year $t$ will survive the following $\Delta n$ years. This probability is defined as follows:

$$
\mathcal{S}_{n}(\Delta n)=E\left(e^{-\int_{t}^{t+\Delta n} h_{\tau} d \tau} \mid \mathcal{F}_{t}\right)
$$

where $\mathcal{F}_{t}$ describes the information at time $t$. We compute formula (38) for various values of $\Delta n, 0 \leq \Delta n \leq 30$ years. For simplicity, this calculation is made by numerically integrating the stochastic differential equations (10)-(12) with the explicit Euler method. We simulate 1500 trajectories to compute the mean value appearing in (38). This is a preliminary study. The same procedure can be used with real data to calibrate the parameters of model (1), (3)-(5).

We start by showing the survival probability $\mathcal{S}_{n}$ as a function of time for various values of the correlation coefficient $\rho_{Y}$. The model parameters appearing in (5) are chosen as in Recchioni and Screpante 2014 (see Table 1 below) except for the values of $\rho_{Y}$ and $\gamma$. The values of these two parameters will be specified later in this section.

Note that $h_{0}$ can be interpreted as the hazard rate at birth and it depends on the observed cohort. Table 1 shows the values of the parameters appearing in (5) corresponding to three cohorts. These parameters are motivated by an empirical analysis presented in Bertocchi et al. 2011 with data from the Italian Human Mortality Database available at http://www.mortality.org/. When $\gamma$ is equal to zero the model defined by equation (1) and (5) reduces to the Milevsky-Promislow model. Here, we choose $a=1$ while $\gamma=0.5, \gamma=1$ and 
Table 1: Parameter values of the demographic component model (1), (3)-(5).

\begin{tabular}{ccccc}
\hline cohort & $h_{0}$ & $b$ & $g$ & $\sigma$ \\
\hline 1966 & 0.0001180 & 0.3338 & 0.0761 & 0.0341 \\
1977 & 0.0001175 & 0.6315 & 0.0722 & 0.0311 \\
1980 & 0.0001431 & 0.5990 & 0.0653 & 0.0455 \\
\hline
\end{tabular}

$\gamma=5$ (see Eqs. (3)-(5)). The values of the parameters appearing in (3)-(4) are those shown in Christoffersen, Heston and Jacob 2009 and reported in Table 2. These values are obtained by calibrating the Heston model to U.S. S\&P 500 index and corresponding call and put option prices. We set $\mu=0.001, x_{0}=0$ and $v_{0}=0.01$.

Table 2: Parameter values of the financial component of model (1), (3)-(5).

\begin{tabular}{ccccc}
\hline year & $\chi$ & $\theta$ & $\epsilon$ & $\rho_{v}$ \\
\hline 2000 & 2.5751 & 0.0678 & 0.6561 & -0.6975 \\
2004 & 1.6048 & 0.0464 & 0.3796 & -0.7670 \\
\hline
\end{tabular}

Figure 1 and Figure 2 show the survival probability as a function of the age of individuals belonging to three cohorts: 1966 (left panel), 1977 (middle panel) and 1980 (right panel) in the case they are living in 2000 (Fig. 1) and in the case they are living in 2004 (Fig. 2). Each panel displays three curves (survival probability versus age) corresponding to three values of the correlation coefficient, $\rho_{Y}, \rho_{Y}=-0.99,0,0.99$. The parameter values of the financial component of the model used to obtain the results in Figures 1-2 are listed in Table 2.
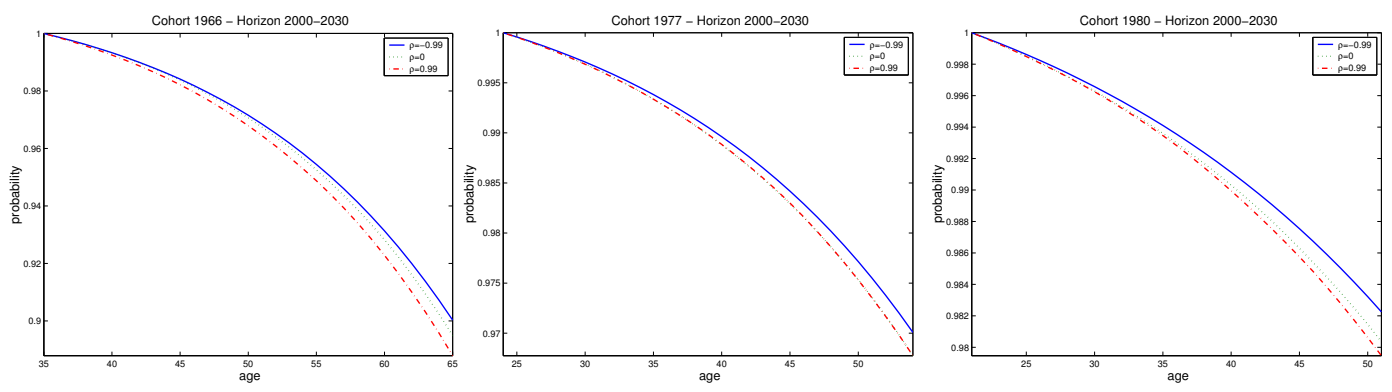

Figure 1: Survival probability as a function of age for individuals belonging to the cohorts 1966 (left panel), 1977 (middle panel), and 1980 (right panel) in the case they are living in 2000. The values of the model parameters are shown in Tables 1 and 2 , while $\gamma=1$.

In order to investigate the impact of parameter $\gamma$, we repeat the experiment above only for cohort 1980 in the case they are living in 2000 using different 

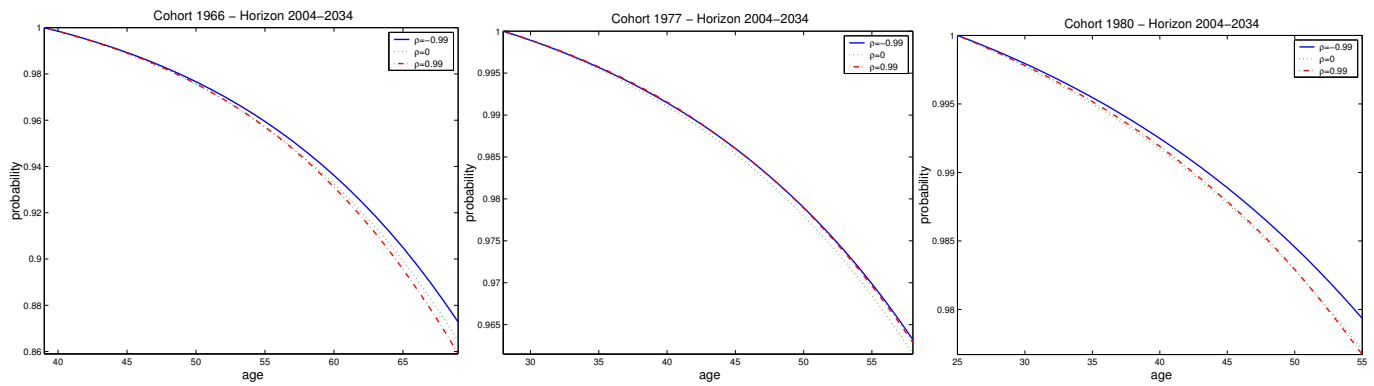

Figure 2: Survival probability as a function of age for individuals belonging to the cohorts 1966 (left panel), 1977 (middle panel) and 1980 (right panel) in the case they are living in 2004. The values of the model parameters are shown in Tables 1 and 2 , while $\gamma=1$.
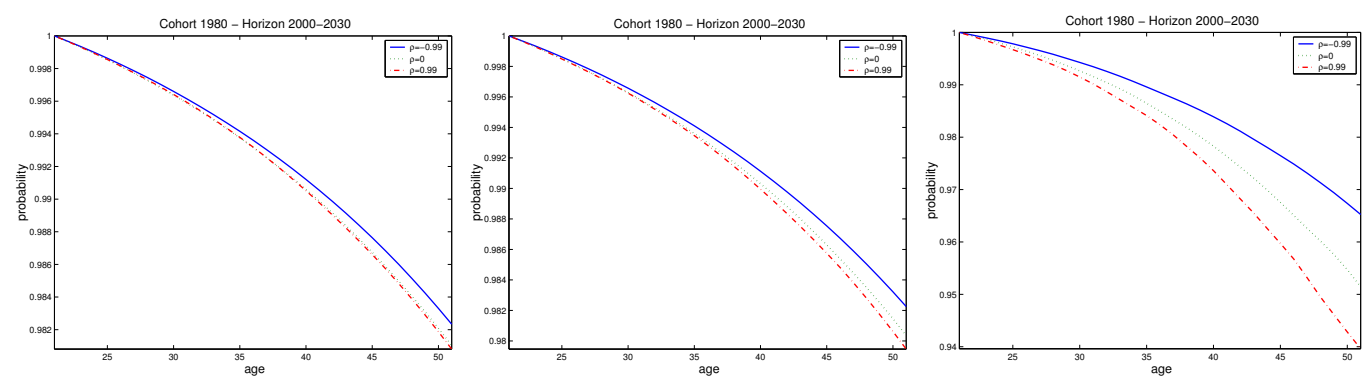

Figure 3: Survival probability as a function of age for individuals belonging to the 1980 cohort should they be living in 2000. The values of the model parameters are shown in Tables 1 and 2, while $\gamma=0.5$ (left panel), $\gamma=1$ (middle panel) and $\gamma=5$ (right panel).

values of $\gamma, \gamma=0.5, \gamma=1$ and $\gamma=5$. The three panels in Figure 3 show the results of this experiment for the three values of $\gamma, \gamma=0.5$ (left panel), $\gamma=1$ (middle) and $\gamma=5$ (right panel).

Three curves are displayed in each panel: a solid line corresponding to $\rho_{Y}=-0.99$, a dotted line corresponding to $\rho_{Y}=0$ and dash-dotted line corresponding to $\rho_{Y}=0.99$. Comparing the curves in the three panels, it is clear that parameter $\gamma$ strongly affects the value of the survival probability. Increasing $\gamma$ from 0.5 to 5 produces significant differences in the values of the survival probability corresponding to different values of the correlation coefficient $\rho_{Y}$. This study shows that the model is able to reproduce a stylized fact. That is, negative values of $\rho_{Y}$ imply larger values of the survival probability. This is due to the fact that decreasing values of $\rho_{Y}$ correspond to decreasing values of the variance $\varphi$ given in (18). Thus, by virtue of Ito's lemma and Eq. (1), this implies decreasing values of the drift of the process $h_{t}$. However, referring to (16) we have a negative correlation between the fundamental market index and mortality rate when $a\left(\gamma+\rho_{Y}\right)<0$. In conclusion, the proposed model appears to be suitable for future investigation regarding the correlated behavior between the survival probability and the market index performance of a given 
country.

\section{References}

[1] M. Abramowitz and I.A. Stegun, Handbook of Mathematical Functions, Dover, New York, 1970.

[2] M.I. Bertocchi, R. Giacometti, S. Ortobelli, A stochastic model for mortality rate on Italian data, Journal of Optimization Theory and Applications, 149 (2011), 216-228. https://doi.org/10.1007/s10957-010-9771-5

[3] E. Biffis, Affine processes for dynamic mortality and actuarial valuations, Insurance: Mathematics and Economics, 37 (2005), no. 3, 443-468. https://doi.org/10.1016/j.insmatheco.2005.05.003

[4] C. Blackburn, M. Sherris, Consistent dynamic affine mortality models for longevity risk applications, Insurance: Mathematics and Economics, 53 (2013), no. 1, 64-73. https://doi.org/10.1016/j.insmatheco.2013.04.007

[5] P. Christoffersen, S. Heston, K. Jacob, The shape and term structure of the index option smirk: Why multifactor stochastic volatility models work so well, Management Science, 55 (2009), 1914-1932.

https://doi.org/10.1287/mnsc.1090.1065

[6] J. Da Fonseca, M. Grasselli, C. Tebaldi, A multifactor volatility Heston model, Quantitative Finance, 8 (2008), no. 6, 591-604.

https://doi.org/10.1080/14697680701668418

[7] M. Dacorogna, M. Cadena, Exploring the Dependence between Mortality and Market Risks, SCOR Paper, 33 (2015), 2-31.

https://doi.org/10.2139/ssrn.2730520

[8] M. M. Denuit, An index for longevity risk transfer, Journal of Computational and Applied Mathematics, 230 (2009), 411-417.

https://doi.org/10.1016/j.cam.2008.12.012

[9] L. Fatone, F. Mariani, M.C. Recchioni, F. Zirilli, An explicitly solvable multi-scale stochastic volatility model: option pricing and calibration problems, Journal of Futures Markets, 29 (2009), no. 9, 862-893. https://doi.org/10.1002/fut.20390

[10] L. Fatone, F. Mariani, M.C. Recchioni, F. Zirilli, The analysis of real data using a multiscale stochastic volatility model, European Financial Management Journal, 19 (2013), no. 1, 153-179.

https://doi.org/10.1111/j.1468-036x.2012.00584.x 
[11] J.P. Fouque, G. Papanicolaou, R. Sircar, K. Solna, Multiscale stochastic volatility asymptotics, Multiscale Modeling and Simulation, 2 (2003), 2242. https://doi.org/10.1137/030600291

[12] E. Luciano, E. Vigna, Mortality risk via affine stochastic intensities: calibration and empirical relevance, Belgian Actuarial Bulletin, 8 (2008), $5-16$.

[13] L. Jalen, R. Mamon, Valuation of contingent claims with mortality and interest rate risks, Mathematical and Computer Modelling, 49 (2009), 18931904. https://doi.org/10.1016/j.mcm.2008.10.014

[14] M.A. Milevsky, S.D. Promislow, Mortality derivatives and the option to annuitise, Insurance: Mathematics and Economics, 29 (2001), 299-318. https://doi.org/10.1016/s0167-6687(01)00093-2

[15] F. Oberhettinger, Fourier Transforms of Distributions and their Inverses: A Collection of Tables, Academic Press, New York, 1973. https://doi.org/10.1016/c2013-0-07466-x

[16] M.C. Recchioni, A. Scoccia, An Analytically Tractable Multi-asset Stochastic Volatility Model, Applied Mathematical Sciences, 8 (2014), no. 27, 1339-1355. https://doi.org/10.12988/ams.2014.311646

[17] M.C. Recchioni, F. Screpante, A hybrid method to evaluate pure endowment policies: Credit Agricole and ERGO Index linked policies, Insurance: Mathematics and Economics, 57 (2014), 114-124. https://doi.org/10.1016/j.insmatheco.2014.05.006

[18] M.C. Recchioni, Y. Sun, An explicitly solvable Heston model with stochastic interest rate, European Journal of Operational Research, 249 (2016), 359-377. https://doi.org/10.1016/j.ejor.2015.09.035

[19] D.F. Schrager, Affine stochastic mortality, Insurance: Mathematics and Economics, 38 (2006), 81-97. https://doi.org/10.1016/j.insmatheco.2005.06.013

\section{Received: August 6, 2016; Published: November 2, 2016}

